\title{
Steady state temperature distribution in a cylindrical electrical conductor-Non-linear effects
}

\author{
B. Berbar, A. Jordan and M. Benmouna \\ Centre Universitaire de Tlemcen, Département de Physique, B.P. 119, Tlemcen, Algeria
}

(Reçu le 8 mars 1983, révisé le 22 juin, accepté le 27 juin 1983)

\begin{abstract}
Résumé. - Nous résolvons l'équation non linéaire de conduction de la chaleur dans un conducteur cylindrique en régime permanent. Nous supposons que la résistivité électrique $\rho$ et la conductivité thermique $\lambda$ sont fonctions linéaires de la température. Nous utilisons une méthode approximative de linéarisation optimale et comparons la solution analytique de cette méthode avec la solution numérique de l'équation non linéaire originale.
\end{abstract}

Abstract. - We solve the non-linear steady state heat conduction equation in a cylindrical conductor. We assume that the electrical resistivity $\rho$ and the thermal conductivity $\lambda$ are both linear functions of the temperature. We use an approximate optimal linearization method and compare the analytical results of this method with the numerical solution of the original non-linear equation.

\section{Introduction.}

The resolution of the heat conduction equation using approximate methods has received particular attention in recents years. This is partially due to the development in the experimental methods of measuring parameters such as the electrical resistivity $\rho$ and the thermal conductivity $\lambda$ as functions of the temperature $T[1,2]$. Knowing the exact variation of $\rho$ and $\lambda$ with temperature, one can determine the steady state temperature distribution by solving the following equation :

$$
\nabla \cdot[\lambda(T) \nabla T]=-q(\mathbf{r}, T)
$$

where $q(\mathbf{r}, T)$ is the rate of internal heat production and $\mathbf{r}$ is the space parameter. This equation has been solved by various authors [3-6] using approximate methods in the case of rectangular coordinates. However, there are several cases of practical interest where one has to solve equation 1 in cylindrical coordinates which is the problem we wish to present in this paper. One can apply the results of these calculations to the problem of heat transfer in electrical equipment using direct current.

Here we solve equation 1 by assuming the heat production is due to the direct current that flows in a cylindrical conductor. The time dependent case will be considered in another paper. This problem has been solved by Hoffer [7] in the case where $\rho$ is a linear function of temperature but $\lambda$ is constant. Here, we assume that both $\rho$ and $\lambda$ are linear functions of the temperature $T$. We choose these forms because they are reasonable in the range of temperature which we consider here, although other forms can be accommodated in our theoretical approach.

\section{Resolution of the steady state heat equation.}

In the case of an infinite, isotropic, cylindrical conductor, equation 1 becomes

$$
\lambda(T)\left[\frac{\mathrm{d}^{2} T}{\mathrm{~d} r^{2}}+\frac{1}{r} \frac{\mathrm{d} T}{\mathrm{~d} r}\right]+\frac{\mathrm{d} \lambda(T)}{\mathrm{d} T}\left(\frac{\mathrm{d} T}{\mathrm{~d} r}\right)^{2}+\rho(T) j^{2}=0
$$

where $T$ is the difference of temperature expressed in $[\mathrm{K}]$ and corresponds to the increase above the ambient temperature $T_{0}, r$ is the radial coordinate, and $j=I / \pi r_{0}^{2}$ is the constant direct current density $\left[\mathrm{A} / \mathrm{m}^{2}\right]$. To solve equation 2 we use the following boundary conditions :

$$
\begin{gathered}
\left.\frac{\mathrm{d} T}{\mathrm{~d} r}\right|_{r=0}=0 \\
\left.\lambda(T) \frac{\mathrm{d} T}{\mathrm{~d} r}\right|_{r=r_{0}}=-\varepsilon T\left(r_{0}\right)
\end{gathered}
$$

where $r_{0}$ is the radius of the conductor and $\varepsilon$ the convective heat transfer coefficient [W $\mathrm{K}^{-1} \mathrm{~m}^{-2}$ ]. In equations 2 and $3 \rho(T)$ and $\lambda(T)$ can be arbitrary functions 
of temperature. In the case where $\lambda$ is constant $\left(\lambda=\lambda_{0}\right)$ and $\rho$ is a linear function of temperature

$$
\rho(T)=\rho_{0}(1+\alpha T) .
$$

Equation 2 becomes

$$
\frac{\mathrm{d}^{2} T}{\mathrm{~d} r^{2}}+\frac{1}{r} \frac{\mathrm{d} T}{\mathrm{~d} r}+\alpha c_{0} T+c_{0}=0
$$

where $c_{0}=\rho_{0} j^{2} / \lambda_{0}\left[\mathrm{~K} \mathrm{~m}^{-2}\right], \rho_{0}$ and $\lambda_{0}$ are the electrical resistivity and the thermal conductivity at $T_{0}$ respectively, and $\alpha$ is a pumerical coefficient. In the latter case the boundary condition $3 b$ becomes simply $\left.\frac{\mathrm{d} T}{\mathrm{~d} r}\right|_{r=r_{0}}=-\frac{\varepsilon}{\lambda_{0}} T\left(r_{0}\right)$.

The solution of equation 5 satisfying these boundary conditions can be obtained as [7]

$T(r)=\frac{\varepsilon}{\alpha} \frac{J_{0}\left(r \sqrt{\alpha c_{0}}\right)}{\varepsilon J_{0}\left(r_{0} \sqrt{\alpha c_{0}}\right)-\lambda_{0} \sqrt{\alpha c_{0}} J_{1}\left(r \sqrt{\alpha c_{0}}\right)}-\frac{1}{\alpha}[\mathrm{K}]$

where $J_{0}(x)$ and $J_{1}(x)$ are Bessel functions of the first kind [8]. This result has also been obtained by Hoffer [7] by solving the transient case and taking the infinite time limit. It is important to note that for this result to be physically acceptable, one has to make sure that the denominator $\varepsilon J_{0}\left(r_{0} \sqrt{\alpha c_{0}}\right)-$ $\lambda_{0} \sqrt{\alpha c_{0}} J_{1}\left(r_{0} \sqrt{\alpha c_{0}}\right)$ remains always positive definite, which introduces a constraint in the choice of the parameters involved in this expression. If one assumes that both $\rho$ and $\lambda$ are linear functions of the temperature, which means that in addition to (4), we have :

$$
\lambda(T)=\lambda_{0}\left(1+\alpha^{\prime} T\right)
$$

where $\alpha^{\prime}$ is a numerical factor. Equation 2 becomes

$$
\begin{aligned}
\left(1+\alpha^{\prime} T\right)\left[\frac{\mathrm{d}^{2} \mathrm{~T}}{\mathrm{~d} r^{2}}+\frac{1}{r} \frac{\mathrm{d} T}{\mathrm{~d} r}\right] & +\alpha^{\prime}\left(\frac{\mathrm{d} T}{\mathrm{~d} r}\right)^{2}+ \\
& +\alpha c_{0} T+c_{0}=0 .
\end{aligned}
$$

This is a non-linear differential equation which does not have an exact analytical solution. To solve this equation we propose to use the method of optimal linearization [4] which consists of approximating equation 8 by a linear equation of the form

$$
\lambda_{\mathrm{op}}\left[\frac{\mathrm{d}^{2} T}{\mathrm{~d} r^{2}}+\frac{1}{r} \frac{\mathrm{d} T}{\mathrm{~d} r}\right]+\rho(T) j^{2}=0
$$

where we have introduced a constant optimal thermal conductivity $\lambda_{\text {op }}$ which will be determined by the procedure described below.

In the present method one replaces $\lambda_{\text {op }}$ by a parameter $k$ and introduces the quantity $I(k)$

$$
I(k)=\int_{0}^{r_{0}} \mathrm{~d} r \Delta^{2},
$$

where $\Delta$ is obtained by taking the difference between equations 9 and 2 :

$$
\Delta=[k-\lambda(T)]\left[\frac{\mathrm{d}^{2} T}{\mathrm{~d} r^{2}}+\frac{1}{r} \frac{\mathrm{d} T}{\mathrm{~d} r}\right]-\frac{\mathrm{d} \lambda(T)}{\mathrm{d} T}\left(\frac{\mathrm{d} T}{\mathrm{~d} r}\right)^{2} .
$$

Combining equations 7, 10 and 11 one can put $I(k)$ in the form

$$
I(k)=A k^{2}-2(B+C) k+D,
$$

where the coefficients $A, B, C$ and $D$ are given by

$$
\begin{aligned}
& A=\int_{0}^{r_{0}} \mathrm{~d} r\left[\frac{\mathrm{d}^{2} T}{\mathrm{~d} r^{2}}+\frac{1}{r} \frac{\mathrm{d} T}{\mathrm{~d} r}\right]^{2} \\
& B=\int_{0}^{r_{0}} \mathrm{~d} r \lambda(T)\left[\frac{\mathrm{d}^{2} T}{\mathrm{~d} r^{2}}+\frac{1}{r} \frac{\mathrm{d} T}{\mathrm{~d} r}\right] \\
& C=\int_{0}^{r_{0}} \mathrm{~d} r \frac{\mathrm{d} \lambda(T)}{\mathrm{d} T}\left(\frac{\mathrm{d} T}{\mathrm{~d} r}\right)^{2}\left[\frac{\mathrm{d}^{2} T}{\mathrm{~d} r^{2}}+\frac{1}{r} \frac{\mathrm{d} T}{\mathrm{~d} r}\right] \\
& D=\int_{0}^{r_{0}} \mathrm{~d} r\left[\left(\frac{\mathrm{d}^{2} T}{\mathrm{~d} r^{2}}+\frac{1}{r} \frac{\mathrm{d} T}{\mathrm{~d} r}\right) \lambda(T)+\left(\frac{\mathrm{d} T}{\mathrm{~d} r}\right)^{2} \frac{d \lambda(T)}{\mathrm{d} T}\right]^{2}
\end{aligned}
$$

$\lambda_{\text {op }}$ is obtained by letting

$$
\frac{\mathrm{d} I(k)}{\mathrm{d} k}=0
$$


whose solution is

$$
k=\lambda_{\mathrm{op}}=\frac{B+C}{A} .
$$

To calculate $\lambda_{\text {op }}$ explicitly one needs an expression for $T(r)$ which is given by equation 6 . This yields :

$$
\lambda_{\mathrm{op}}=\lambda_{0}\left(1+\alpha^{\prime} E\right)
$$

where $E$ is given by

$$
E=F \frac{\int_{0}^{r_{0}} \mathrm{~d} r\left[J_{0}^{2}\left(r \sqrt{\alpha c_{0}}\right)-J_{1}^{2}\left(r \sqrt{\alpha c_{0}}\right)\right] J_{0}\left(r \sqrt{\alpha c_{0}}\right)}{\int_{0}^{r_{0}} \mathrm{~d} r J_{0}^{2}\left(r \sqrt{\alpha c_{0}}\right)}
$$

and

$$
F=\frac{\varepsilon}{\alpha\left[\varepsilon J_{0}\left(r_{0} \sqrt{\alpha c_{0}}\right)-\lambda_{0} \sqrt{\alpha c_{0}} J_{1}\left(r_{0} \sqrt{\alpha c_{0}}\right)\right]} .
$$

Therefore, within the method of optimal linearization, where both $\rho$ and $\lambda$ are linear functions of $T$, the temperature distribution is approximately obtained from the linear equation

$$
\frac{\mathrm{d}^{2} T}{\mathrm{~d} r^{2}}+\frac{1}{r} \frac{\mathrm{d} T}{\mathrm{~d} r}+\frac{\rho(T)}{\lambda_{\mathrm{op}}} j^{2}=0
$$

with the new boundary condition

$$
\left.\frac{\mathrm{d} T}{\mathrm{~d} r}\right|_{r=r_{0}}=-\frac{\varepsilon}{\lambda_{\mathrm{op}}} T\left(r_{0}\right) .
$$

The solution of equation 17 satisfying the boundary conditions $3 a$ and 18 is

$$
T(r)=\frac{\varepsilon}{\alpha} \frac{J_{0}\left(r \sqrt{\alpha c_{0}^{\prime}}\right)}{\varepsilon J_{0}\left(r_{0} \sqrt{\alpha c_{0}^{\prime}}\right)-\lambda_{\mathrm{op}} \sqrt{\alpha c_{0}^{\prime}} J_{1}\left(r_{0} \sqrt{\alpha c_{0}^{\prime}}\right)}-\frac{1}{\alpha}[\mathrm{K}]
$$

where $\quad c_{0}^{\prime}=\frac{c_{0}}{1+\alpha^{\prime} E}$.

\section{Results.}

In figure 1, we have plotted curve 1 corresponding to the temperature distribution $T(r)$ as given by equation 19 using [7]

$$
\begin{aligned}
r_{0} & =5 \times 10^{-3}[\mathrm{~m}] \\
I & =200[\mathrm{~A}] \\
\rho_{0} & =2.9 \times 10^{-8}[\Omega \mathrm{m}], \\
\lambda_{0} & =180\left[\mathrm{~W} \mathrm{~m} \mathrm{~W}^{-1} \mathrm{~K}^{-1}\right], \quad \mathrm{Al} \text { at } 293[\mathrm{~K}] \\
\alpha & =43 \times 10^{-4}\left[\mathrm{~K}^{-1}\right] \\
\alpha^{\prime} & =-3.4 \times 10^{-4}\left[\mathrm{~K}^{-1}\right] \\
\varepsilon & =16.6\left[\mathrm{~W} \mathrm{~m}^{-2} \mathrm{~K}^{-1}\right] .
\end{aligned}
$$

Obviously the value of $\alpha^{\prime} E$ obtained by using these parameters is so small $(-0.01096)$ that one does not expect to find any significant deviation from the linear case where $\lambda=\lambda_{0}$. We have also plotted in the same figure curves 2 and 3 corresponding to $\alpha^{\prime} E=+0.5$ and -0.5 , respectively. These numbers may not correspond to real cases and are chosen in order to enhance artificially the effect of non linearity. We have also solved numerically the non-linear equation 2 by applying a Runge-Kutta method of the 4th order and using HP 9825B calculator. 


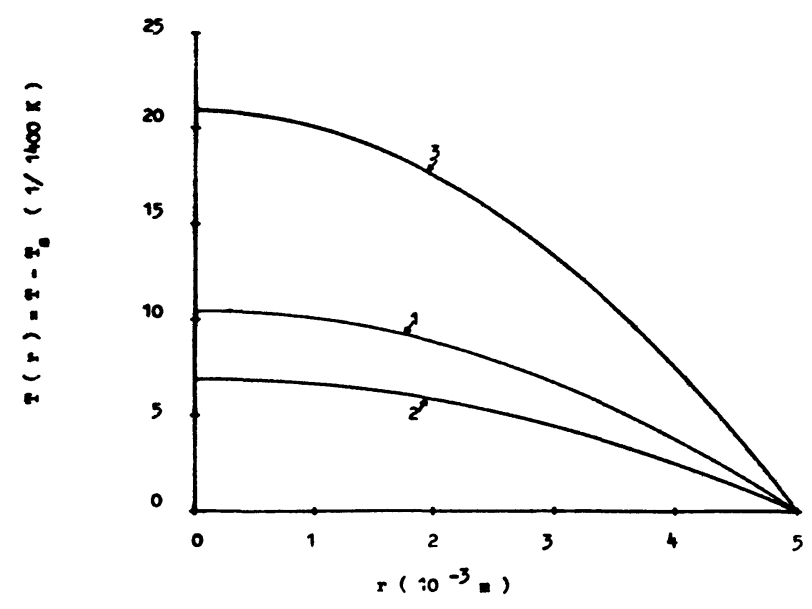

Fig. 1. - The variation of the temperature as a function of $r$ as given by (8) and (9), using $I=200$ [A] and $\varepsilon=16.6$ $\left[\mathrm{W} \mathrm{m}^{-2} \mathrm{~K}^{-1}\right]$ : curve $1: \alpha^{\prime}=-3.4 \times 10^{-4}\left[\mathrm{~K}^{-1}\right]$; curve $2: \alpha^{\prime} E=+0.5$; curve $3: \alpha^{\prime} E=-0.5$.

For the sake of comparison with the approximate solution given by equation 19 we have used the parameters displayed by (20) except for $\alpha^{\prime}$ which is taken to be $-3.4 \times 10^{-4}\left[\mathrm{~K}^{-1}\right]$ for curve 1 , and $\pm 0.5 / E$ for curves 2 and 3, respectively. We obtain a good agreement between the exact numerical solution and the approximate analytical solution, to within an accuracy of $10^{-4}[\mathrm{~K}]$.

The degree of accuracy of our optimal linearization method is a function of the temperature level within the conductor, or equivalently on the choice of the parameters such as $I$ and $\varepsilon$. To show this dependence, we have plotted the curves in figure 2 choosing the same parameters as in figure 1, except for $I$ and $\varepsilon$ which are taken to be $I=2000[\mathrm{~A}]$ and $\varepsilon=300\left[\mathrm{~W} \mathrm{~m} \mathrm{~m}^{-2} \mathrm{~K}^{-1}\right]$.

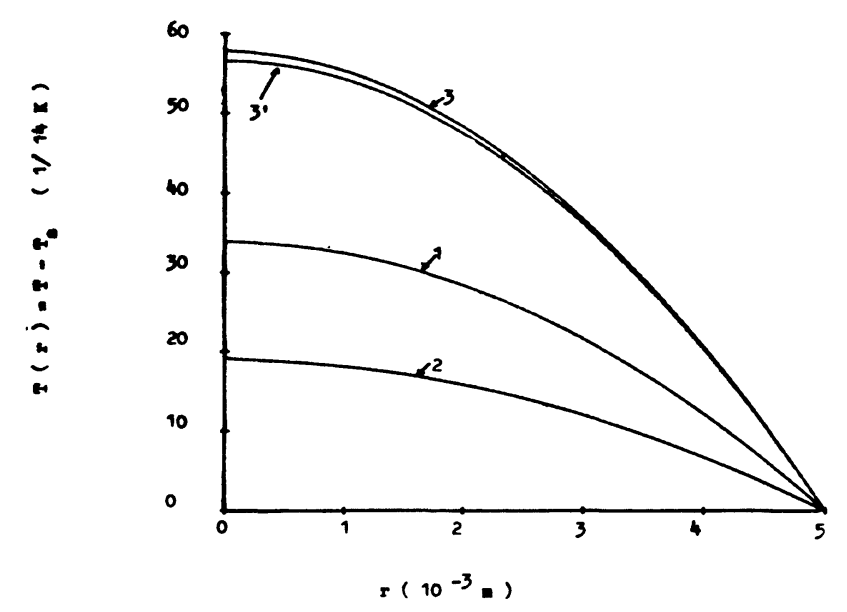

Fig. 2. - The variation of the temperature as a function of $r$ as given by (8) and (9), using $I=2000$ [A] and $\varepsilon=300$ $\left[\mathrm{W} \mathrm{m} \mathrm{m}^{-2} \mathrm{~K}^{-1}\right]$ : curve $1: \alpha^{\prime}=-3.4 \times 10^{-4}\left[\mathrm{~K}^{-1}\right]$; curve $2: \alpha^{\prime} E=+0.5$; curve $3: \alpha^{\prime} E=-0.5$ (Eq. 8); curve 4 : $\alpha^{\prime} E=-0.5$ (Eq. 9).

This shows that the optimal linearization method which we have presented here can be used to solve the non-linear steady state heat conduction equation with a fairly high degree of accuracy.

It can be particularly useful when $\lambda$ depends strongly on temperature, i.e. when $\alpha^{\prime}$ is large. However, this method is not unique to the case of electrical conductors but can be generalized to other heat conducting systems.

Furthermore, one observes that the temperature is practically constant within the conductor, but it depends strongly on other parameters such as the electrical current $I$ and the heat exchange parameter $\varepsilon$. For the sake of simplicity, we investigate the effects of these parameters on the temperature at the centre $T_{\mathrm{m}}=T(r=0)$, which is given by

$$
T_{\mathrm{m}}=\frac{\varepsilon}{\alpha} \frac{1}{\varepsilon J_{0}\left(r_{0} \sqrt{\alpha c_{0}^{\prime}}\right)-\lambda_{\mathrm{op}} \sqrt{\alpha c_{0}^{\prime}} J_{1}\left(r_{0} \sqrt{\alpha c_{0}^{\prime}}\right)}-\frac{1}{\alpha}[\mathrm{K}]
$$


Table I.1. - The coefficient $\varepsilon$ characterizing the rate of heat exchange using various stationary fluids [9].

\begin{tabular}{|c|c|c|c|c|c|}
\hline \multirow[b]{2}{*}{ Fluid } & \multirow[b]{2}{*}{$\begin{array}{l}\text { Air (Conductor } \\
\text { with polished } \\
\text { surface) }\end{array}$} & \multirow[b]{2}{*}{$\begin{array}{l}\text { Air (Conductor } \\
\text { with black } \\
\text { surface) }\end{array}$} & \multirow[b]{2}{*}{ Oil } & \multirow[b]{2}{*}{ Water } & \multirow[b]{2}{*}{ Boiling water } \\
\hline & & & & & \\
\hline- & - & - & - & - & - \\
\hline$\left[\mathrm{W} \mathrm{m} \mathrm{m}^{-2} \mathrm{~K}^{-1}\right]$ & 8 & 20 & 300 & 2300 & 4000 \\
\hline
\end{tabular}

Table I.2. - The coefficient $\varepsilon$ characterizing the rate of heat exchange in electrical equipment using air-flow at various speeds [9].

$\begin{array}{crrrrrr}\text { Speed }\left[\mathrm{ms}^{-1}\right] & 0 & 1 & 5 & 10 & 20 & 50 \\ \varepsilon\left[\mathrm{W} \mathrm{m}^{-2} \mathrm{~K}^{-1}\right] & 20 & 25 & 44 & 70 & 110 & 150\end{array}$

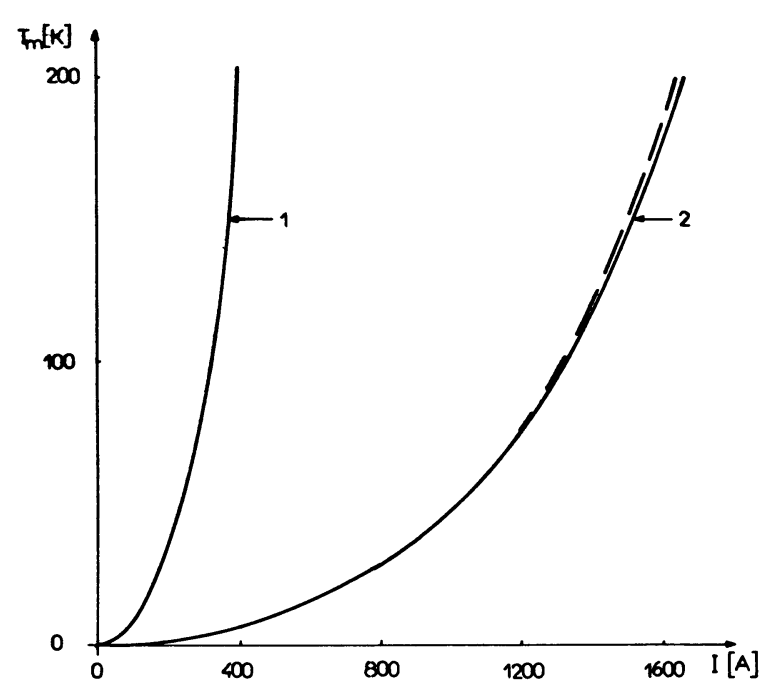

Fig. 3. - The variation of $T_{\mathrm{m}}$ as a function of the current $I$ in the case where $\lambda=\lambda_{0}:$ curve $1: \varepsilon=16.6\left[\mathrm{~W} \mathrm{~m}^{-2} \mathrm{~K}^{-1}\right]$; curve $2: \varepsilon=300\left[\mathrm{~W} \mathrm{~m}^{-2} \mathrm{~K}^{-1}\right]$. The dotted line corresponds to the case where $\lambda$ is a linear function of the temperature with $\alpha^{\prime} E=-0.5$ and $\varepsilon=300\left[\mathrm{~W} \mathrm{~m}^{-2} \mathrm{~K}^{-1}\right]$.

In figure 3, we have plotted $T_{\mathrm{m}}$ as a function of the current $I$ using the following parameters [7],

$$
\begin{aligned}
& \rho_{0}=2.9 \times 10^{-8}[\Omega \mathrm{m}] \\
& \alpha=43 \times 10^{-4}\left[\mathrm{~K}^{-1}\right] \\
& \lambda_{0}=180\left[\mathrm{~W} \mathrm{~m}^{-1} \mathrm{~K}^{-1}\right] .
\end{aligned}
$$

Curve 1 corresponds to $\varepsilon=16.6\left[\mathrm{~W} \mathrm{~m} \mathrm{~m}^{-2} \mathrm{~K}^{-1}\right]$ and has been taken from reference 7. Curve 2 is plotted for $\varepsilon=300\left[\mathrm{~W} \mathrm{~m}^{-2} \mathrm{~K}^{-1}\right]$ (see tables I).

One observes that $\varepsilon$ affects strongly the temperature in the conductor.

The effect of $\varepsilon$ on $T_{\mathrm{m}}$ when $\lambda$ is a linear function of temperature is not significantly different from the limit when $\lambda=\lambda_{0}$, even by letting $\alpha^{\prime} E=-0.5$ (see dotted curve in Fig. 3).

\section{Conclusions.}

In this paper, we have applied the method of optimal linearization to solve the steady state non-linear heat conduction equation for a cylindrical conductor. The analytical results of the linear " optimal " equation have been compared with the numerical resolution of the exact non-linear equation, and the agreement was found to be good. Here, we have chosen the particular case where the parameters $\rho(T)$ and $\lambda(T)$ vary linearly with temperature, but the method of optimal linearization is not restricted to these types of variations only.

\section{Acknowledgments.}

We thank Dr. E. Benoit for his help in the numerical calculations.

\section{References}

[1] TAYLOR, R. E., Thermal Properties of Tungsten SRM'S 730 and 799, Trans. ASME, 100 (1978) 330-333.

[2] Farnia, K., BeCK, J. V., Numerical Solution of Transient Heat Conduction Equation for Heat Treatable alloys whose Thermal Properties change with Time and Temperature, J. Heat Transfer (1977) 471-478.

[3] YANG, K. T., Transient Conduction in a Semi-Infinite Solid with Variable Thermal Conductivity, J. Appl. Mech. 25 (1958) 146-147.

[4a] Vujanovic, B. and Baclic, B., Applications of Gauss Principle of Least Constraint to the Non-Linear Heat-Transfer Problem, Int. J. Heat Mass Transfer 19 (1976) 721-730.

[4b] Vujanovic, B., Application of the Optimal Linearization Method to the Heat Transfer-Problem, Int.
J. Heat Mass Transfer 16 (1973) 1111-1117.

[5] TAler, J., Application of Gauss's Principle of Least Constraint to Approximate Solution of Heat Conduction Equation, Eng. Trans. 25 (1977) 349-368.

[6] Aguirre-Ramirez, G. et Oden, J. T., Finite Element Technique applied to Heat Conduction in Solids with Temperature dependent Thermal Conductivity, Int. J. Numer. Meth. Eng. 7 (1973) 345-355.

[7] HOFFER, O., Instationäre Temperaturverteilung in Einem Runddraht, Archiv Elektrotech. 60 (1978) 319-325.

[8] Abramovitz, M. and Stegun, I. A., Handbook of Mathematical Functions AMS 555, NBS (1964).

[9] Moon, P. et SPEncer, D. E., Field Theory for Engineers, (Van Nostrand, New York) 1961. 